\title{
Noninvasive prenatal test for fetal chromosomal aneuploidies by massively parallel sequencing of cell- free fetal DNA in maternal plasma: The first clinical experience in Korea
}

\author{
Sung-Hee Han, Young-Ho Yang*, Jae-Song Ryu, Myung-Soo Kang, Young-Jin Kim, and Kyoung-Ryul Lee
} Division of Prenatal Molecular Genetics, Department of Laboratory Medicine, Seoul Clinical Laboratories, Yongin, Korea

\begin{abstract}
Purpose: Noninvasive prenatal test (NIPT) by massively parallel sequencing (MPS) of cell-free fetal DNA in maternal plasma marks a significant advancement in prenatal screening, minimizing the need for invasive testing of fetal chromosomal aneuploidies. Here, we report the initial clinical performance of NIPT in Korean pregnant women.

Materials and Methods: MPS-based NIPT was performed on 910 cases; $5 \mathrm{~mL}$ blood samples were collected and sequenced in the Shenzhen BGI Genomic Laboratory to identify aneuploidies. The risk of fetal aneuploidy was determined by L-score and t-score, and classified as high or low. The NIPT results were validated by karyotyping for the high-risk cases and neonatal follow-up for low-risk cases.

Results: NIPT was mainly requested for two clinical indications: abnormal biochemical serum-screening result (54.3\%) and advanced maternal age (31.4\%). Among 494 cases with abnormal biochemical serum-screening results, NIPT detected only 9 (1.8\%) high-risk cases. Sixteen cases (1.8\%) of 910 had a high risk for aneuploidy: 8 for trisomy 21, 2 for trisomy 18, 1 for trisomy 13, and 5 for sex chromosome abnormalities. Amniocentesis was performed for 7 of these cases (43.8\%). In the karyotyping and neonatal data, no false positive or negative results were observed in our study.

Conclusion: MPS-based NIPT detects fetal chromosomal aneuploidies with high accuracy. Introduction of NIPT as into clinical settings could prevent about $98 \%$ of unnecessary invasive diagnostic procedures.
\end{abstract}

Key words: Noninvasive prenatal test (NIPT), Cell-free fetal DNA (cffDNA), Fetal chromosomal aneuploidies.

\section{Introduction}

Prenatal screening and the diagnosis of fetal chromosomal aneuploidies have been essential parts of obstetric care. Conventional prenatal screening for chromosomal aneuploidies, mostly trisomy 21, relies exclusively on biochemical and sonographic measurements performed in the first and second trimesters. With a 3-5\% false-positive rate, first-trimester screening achieves a detection rate of about $60-95 \%$ for trisomy 21 [1-3]. In 1997, Lo et al. [4] first demonstrated the presence

Received: 12 October 2015, Revised: 19 November 2015, Accepted: 19 November 2015, Published: 31 December 2015

${ }^{*}$ Corresponding author: Young-Ho Yang, M.D., Ph.D.

Division of Prenatal Molecular Genetics, Department of Laboratory Medicine, Seoul Clinical Laboratories, 13 Heungdeok 1-ro, Giheung-gu, Yongin 16954, Korea.

Tel: +82-2-6322-1080, Fax: +82-2-790-6509, E-mail:yhyang102@scllab.co.kr

Han SH and Yang YH contributed equally to this work.

This article was presented at the 100st conference of Korean Society of Obstetrics and Gynecology (September 26, 2014).

Conflict of interest: The authors declare that they do not have any conflicts of interest.

(c) This is an open-access article distributed under the terms of the Creative Commons Attribution Non-Commercial License (http://creativecommons.org/licenses/by-nc/4.0/) which permits unrestricted non-commercial use, distribution, and reproduction in any medium, provided the original work is properly cited.

(c) Copyright 2015 by the Korean Society of Medical Genetics 
of circulating cell-free fetal DNA (cffDNA) in maternal plasma, which has opened a new approach to noninvasive prenatal test (NIPT).

Since the introduction of NIPT for fetal chromosomal aneuploidies by massively parallel sequencing (MPS), the clinical application of NIPT for screening high-risk pregnant women has grown significantly. Several validation studies of high-risk populations have demonstrated the detection rates for trisomy 21 of $>99 \%$, 98\% for trisomy 18 , and $89 \%$ for trisomy 13 , with false positive rates of $0.1 \%, 0.1 \%$, and $0.4 \%$, respectively [5-14]. Consequently, in high-risk populations, NIPT has been shown to have a higher sensitivity and specificity than biochemical serumscreening tests that are currently in use for these trisomies. Recently, this new approach to fetal aneuploidy screening has been introduced in routine clinical practice [15].

The excellent performance of NIPT in multiple clinical validations [5-14], the noninvasive nature of the testing, and the positive opinions of many professional societies, including the American Congress of Obstetrics and Gynecology (ACOG), the Society of Maternal and Fetal Medicine (SMFM) [16], the National Society of Genetic Counselors (NSGC) [17], and the International Society of Prenatal Diagnosis (ISPD) [18], have resulted in its adoption by many clinical institutions. The clinical introduction of NIPT in the United States has resulted in a marked decline in the number of invasive procedures of about 17\%, while in the United Kingdom and Australia the decline was more gradual, with an average of $1.8-8 \%$ per year [19-22]. In

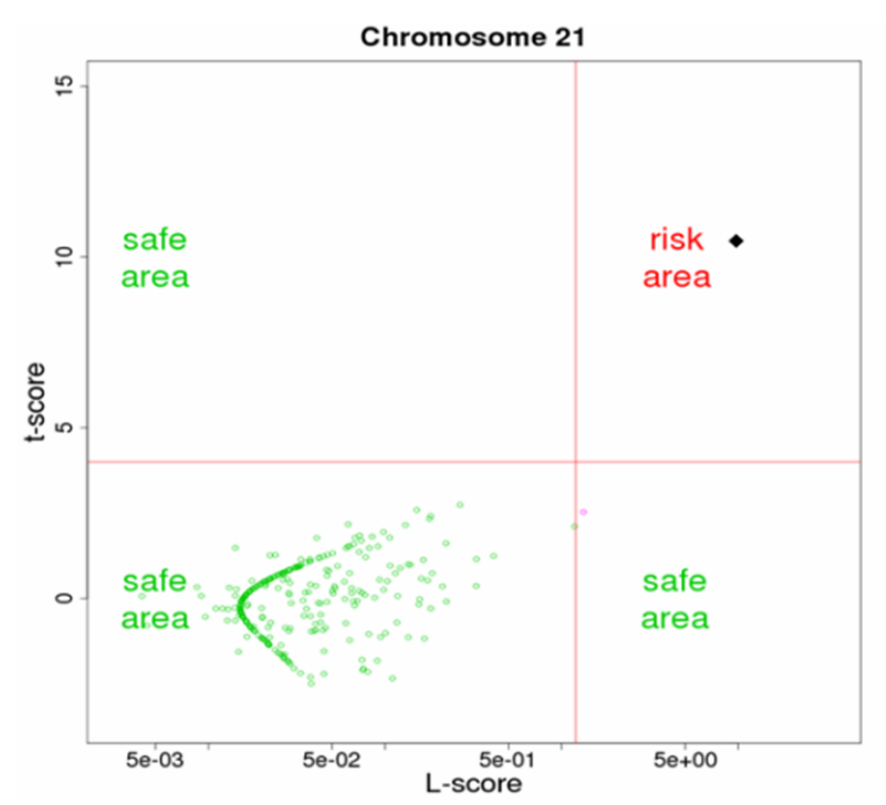

a recent survey, around $86 \%$ of physicians in the United States claimed they would offer NIPT to high-risk women within 1 year after the survey [21]. In Hong Kong, a study on pregnant women showed that about $98 \%$ of invasive diagnostic procedures would be avoided if NIPT were used [5]. In Korea, NIPT was first offered in 2012 by our institution, and with the approval from the Korea Food and Drug Administration in the near future, NIPT can be considered for routine clinical use by local Korean health authorities. As the first institution to offer NIPT for the detection of fetal chromosomal aneuploidies in Korea, we have been able to accrue the clinical experience population dataset to date by including samples from various medical sites. Here, we report the initial clinical performance of NIPT in detecting fetal chromosomal aneuploidies, especially trisomies 21, 18, and 13, in singleton pregnancies in Korea.

\section{Materials and Methods}

For this study, data were acquired from 910 patients from various medical sites in Korea, between May 2, 2012 and December 31, 2013. NIPT was offered to the participants as either a primary or secondary screening. Multiple pregnancies were excluded. A patient with any of the following factors was classified as having a high risk for aneuploidy: advanced maternal age ( $\geq 35$ years); abnormal biochemical serumscreening results, including for the first trimester, sequential, integrated, or quadruple screening; abnormal sonographic

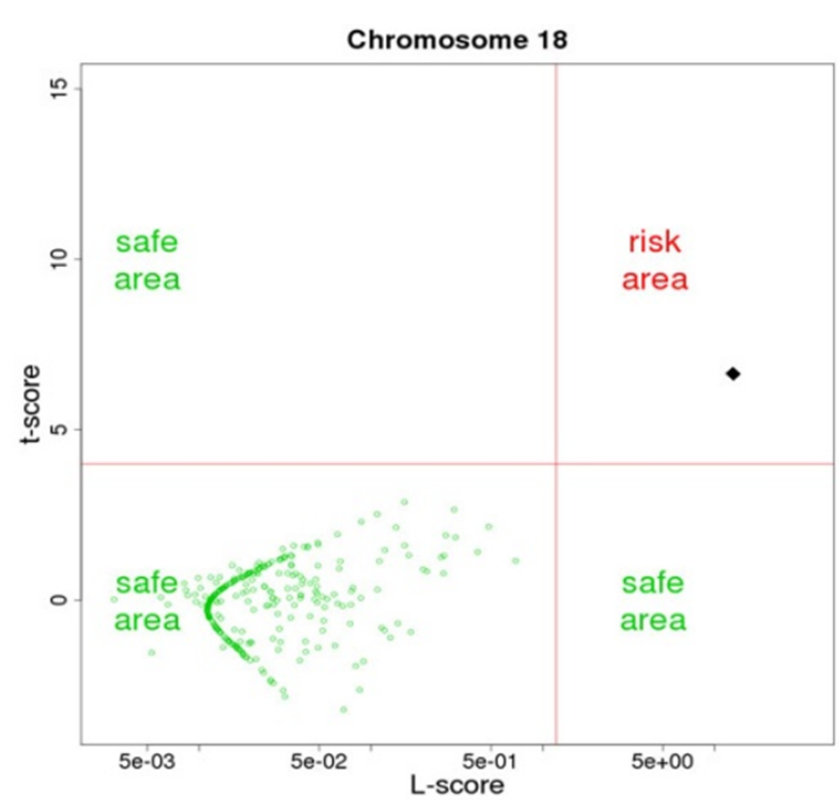

Fig. 1. Identification of fetal aneuploidy by the massively parallel sequencing-based noninvasive prenatal test. The risk of fetal aneuploidy was described by the $\mathrm{L}$-score ( $\mathrm{x}$-axis) and $\mathrm{t}$-score ( $\mathrm{y}$-axis). The high-risk zone is defined by L-score $>1$ and t-score $>2.5$. 
markers; a family history of aneuploidy; or a previous pregnancy with a trisomic fetus. Pretest counseling, including detailed information on the value and limitations of NIPT, was provided for each pregnant woman by an obstetrician. Approval for the study was provided by the ethical committee of Seoul Clinical Laboratories (SCL-IRB-201202).

Five milliliters of peripheral blood were collected into EDTA tubes from each participant, and samples were prepared for cellfree DNA sequencing, as described previously [23]. To extract the plasma, the blood samples were centrifuged twice (at 1,600 g and at $16,000 \mathrm{~g}$ ) within $8 \mathrm{~h}$ of blood collection. Plasma samples were then frozen and sent via courier to the Ministry of Healthaccredited and ISO/IEC17025-certified clinical laboratories of BGI-Health (Shenzhen, China). For each case, the following information was provided by filling out an application form: the date of collection, maternal age, duration of pregnancy, mode of conception, single or multiple pregnancy, indication for NIPT, results of the biochemical serum-screening test, and familial genetic diseases. Upon institutional review board approval, a retrospective chart review was conducted to collect clinical data on those patients who had undergone NIPT. The risk of fetal aneuploidy was determined by the L-score and t-score and classified as high or low. Risk scores represented as a calculated probability for trisomies 21,18, and 13 are provided in the results of the report (Fig. 1). Analyses for sex chromosome abnormalities (SCAs) were performed in this test. If there were suspicions of SCAs, such additional findings were included as 'additional findings' in the results.

Each participant received post-genetic counseling after NIPT by obstetricians. Patients with high-risk results were advised to undergo invasive testing for a prenatal diagnosis. Fetal karyotyping analyses were performed at our institution by conventional G-banded cytogenetic studies. Complete followup was considered once the invasive testing was performed or neonatal data were obtained.

\section{Results}

From May 2, 2012 to December 31, 2013, 910 singleton pregnancies were drawn for prenatal testing with NIPT. On average, NIPT patients were 35.3 years of age at the time of testing (range, 22-46 years). About 61.8\% were 35 years or older. In terms of the parity of 910 patients, the frequency of nullipara and multipara was similar, with a rate of $48.8 \%$ and $51.2 \%$, respectively. The mean gestational age at the time of blood sampling was 16.6 weeks (range, 11-25 weeks). About two thirds
( $n=625,68.7 \%$ ) of the patients were tested at 17 to 20 weeks of gestation. The vast majority (94.3\%) of samples were collected during the second trimester (Table 1).

Risk scores from the NIPT of the first blood samples were provided in 897 (98.6\%) of the 910 pregnancies. Repeated blood sampling was required in 21 cases $(2.3 \%)$ due to problems with blood collection and delivery to the laboratory $(n=9 ; 1$ case of a hemolyzed sample and 8 cases of sample thawing due to transport delay) or assay failure ( $n=12 ; 3$ cases of cell-free DNA extraction failure and 9 cases of analysis failure due to low fetal fraction). In 16 of the 21 cases with no results in the first sampling, further blood samples were obtained and NIPT results with a risk score were provided in 14 cases. Ultimately, 2 samples $(0.2 \%)$ failed to provide informative results and were classified as test failures, which were performed by invasive diagnostic test and could be confirmed a normal karyotype. In the remaining 5 cases with no results in the first sampling, repeated blood sampling was cancelled by the patients after counseling with their obstetricians. Three cases were confirmed to have a normal karyotype by invasive diagnostic procedures. The other two cases were lost to follow-up.

The mean time from blood collection to test results was 12.7 calendar days (range, 11-18 days). The main clinical indication

Table 1. Clinical characteristics of 910 pregnant women who underwent noninvasive prenatal test

\begin{tabular}{|cc|}
\hline \multicolumn{1}{c}{ Clinical characteristic } & Data \\
\hline Maternal age (yr) & $35.3 \pm 4.1(22-46)$ \\
\hline $18-24$ & $2(0.2)$ \\
$25-29$ & $79(8.7)$ \\
$30-34$ & $267(29.3)$ \\
$35-39$ & $431(47.4)$ \\
$\geq 40$ & $131(14.4)$ \\
\hline Parity (nullipara / multipara) & $444(48.8) / 466(51.2)$ \\
\hline Pregnancy by assisted reproductive techniques, & $3(0.3)$ \\
IVF & $16.6 \pm 2.2(11-25)$ \\
\hline Gestational age (wk) at the blood sampling & $15(1.6)$ \\
\hline $9-12$ & $248(27.3)$ \\
\hline $13-16$ & $620(68.1)$ \\
\hline $17-20$ & $23(2.5)$ \\
\hline $21-24$ & $4(0.4)$ \\
\hline 25 & \\
\hline Prior biochemical serum screening test & $494(54.3)$ \\
\hline Yes - screening positive & $38(4.2)$ \\
\hline Yes - screening negative & $378(41.5)$ \\
\hline No &
\end{tabular}

Values are presented as mean \pm standard deviation (range) or number (\%). IVF, in vitro fertilization. 
for testing was abnormal biochemical serum-screening results in 54.3\% (494/910) of pregnancies, followed by advanced maternal age in 31.4\% (286/910) (Fig. 2). In particular, among 494 cases with abnormal biochemical serum-screening results, NIPT high-risk results were detected in only 9 cases (1.8\%).

The description of NIPT high-risk cases is shown in Table 2. In 16 cases, there was a high risk for aneuploidy: in 8 for trisomy 21 (T21), in 2 for trisomy 18 (T18), in 1 for trisomy 13 (T13), and in 5 in sex chromosomes ( 2 cases of $X X Y, 1$ of XYY, 1 of XXX, and 1 of monosomy $X$ ). All 16 high-risk cases were not confirmed by conventional karyotyping, but 7 out of the 16 cases (43.8\%) were confirmed by invasive procedures. Seven patients with high-risk results declined the subsequent fetal karyotyping. Most patients with a high risk of sex chromosome aneuploidy

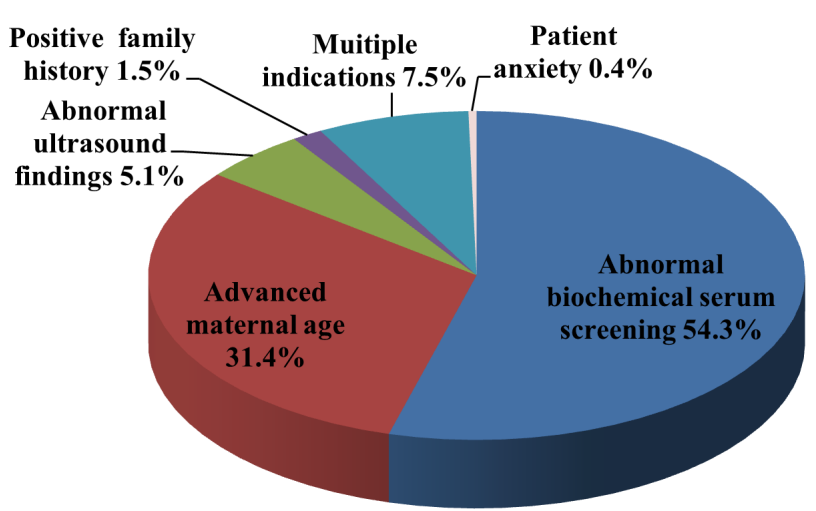

Fig. 2. Clinical indications for noninvasive prenatal test. declined the invasive test. Eight cases with NIPT-high risk results of T21 were validated in 4 cases through prenatal karyotyping with no false results. No karyotype analysis was conducted on 4 patients with high-risk results for T21: one (case 4) with intrauterine fetal demise after blood sampling for NIPT and three refusing invasive procedures for karyotype analysis. Both high-risk cases of T18 were correctly identified through prenatal karyotyping via amniocentesis. One high-risk case of T13 had a spontaneous miscarriage without karyotype confirmation. Outcome data and detailed information for each newborn were available for 894 cases with low risk results in the study. Among them, no women gave birth to a newborn with T21, T18, or T13. Among the 894 follow-ups and 7 cases with karyotyping results, the estimated sensitivity and specificity were 100\%.

\section{Discussion}

This is the first report of Korean clinical experiences using NIPT for the detection of fetal chromosomal aneuploidies. No significant technical or logistic problem was encountered with the introduction of NIPT. However, since this was a new test, clinicians had many questions on its scientific basis, technical details, and clinical performance. Given the very high clinical sensitivity and specificity of NIPT, probably the most important and most common misunderstanding was that they would not need a diagnostic test if NIPT were positive. We have conducted a series of educational presentations for clinicians since the test

Table 2. Clinical characteristics of high-risk cases determined by noninvasive prenatal test $(n=16)$

\begin{tabular}{|c|c|c|c|c|c|}
\hline Case No. & Age (yr) & Duration of gestation & Clinical indications & NIPT result & Karyotyping \\
\hline Case 1 & 36 & 12 weeks 3 days & AMA & High risk T21 & Declined \\
\hline Case 2 & 29 & 19 weeks 1 day & Quad-Down 1:5 & High risk T21 & Trisomy 21 \\
\hline Case 3 & 31 & 13 weeks 4 days & Abnormal US & High risk T21 & Declined \\
\hline Case 4 & 41 & 18 weeks 6 days & Quad-Down 1:5 & High risk T21 & Abortion \\
\hline Case 5 & 31 & 17 weeks & Abnormal US & High risk T21 & Trisomy 21 \\
\hline Case 6 & 33 & 16 weeks & Quad-Down 1:5 & High risk T21 & Trisomy 21 \\
\hline Case 7 & 34 & 18 weeks 4 days & Quad-Down 1:5 & High risk T21 & Trisomy 21 \\
\hline Case 8 & 40 & 17 weeks 5 days & Quad-Down 1:5 & High risk T21 & Declined \\
\hline Case 9 & 38 & 13 weeks & AMA & High risk T18 & Trisomy 18 \\
\hline Case 10 & 29 & 18 weeks 6 days & Edward 1:5 & High risk T18 & Trisomy 18 \\
\hline Case 11 & 38 & 16 weeks 3 days & Quad-Down 1:25 & High risk T13 & Abortion \\
\hline Case 12 & 42 & 17 weeks 1 day & AMA & $X X X$ & Declined \\
\hline Case 13 & 38 & 17 weeks 2 days & AMA & $X X Y$ & Declined \\
\hline Case 14 & 34 & 18 weeks & Quad-Down 1:76 & $X X Y$ & Declined \\
\hline Case 15 & 25 & 16 weeks 6 days & Quad-Down 1:110 & $X Y Y$ & Declined \\
\hline Case 16 & 34 & 17 weeks 1 day & Abnormal US & Monosomy X & Monosomy X \\
\hline
\end{tabular}

AMA, advanced maternal age; US, ultrasound findings. 
was introduced.

Failure to obtain results at the time of the first blood sampling, which occurred in $2.3 \%$ of our cases, was due to problems with blood collection and delivery to the laboratory in one half of the cases and due to assay failure in the other half. Currently, NIPT is performed in a few global laboratory companies, and problems arising from the overseas delivery of samples may be inescapable. In particular, sample delivery problems occurred within three months after the introduction of NIPT. A repeated sample is very likely to provide results in most cases (14/16, 87.5\%). Only two cases with no results in the first sampling had no results the second time around due to assay failure caused by low fetal DNA concentration.

The detection rate for trisomies 21, 18, and 13 was 100\%, which is in line with other international studies [5-14]. No false positive or negative cases were detected in our study. Therefore, we conclude that NIPT is a powerful testing tool in prenatal care. We found 16 cases with high-risk results. All of them, including SCAs, were classified as high-risk for aneuploidy. In addition, we did not detect cases with high-risk results in the low-risk group for aneuploidy. The ACOG and SMFM [16] recommended NIPT as a screening option for women with an increased risk of fetal aneuploidy. This population was defined as women 35 years or older, fetuses with ultrasonographic findings indicative of an increased risk of aneuploidy, women with a history of trisomyaffected offspring, parents carrying a balanced robertsonian translocation with an increased risk of trisomy 13 or trisomy 21, and women with positive first-trimester or second-trimester screening-test results. According to the latest opinion from ACOG [24], given the performance of conventional screening methods and the limitations of NIPT, conventional screening methods remain the most appropriate choice for first-line screening for most women in the general obstetric population.

The whole-genome approach enabled the detection of other chromosomal abnormalities. Most of these additional abnormalities were SCAs. The incidence of SCAs in our study was $5(0.5 \%)$ out of 910, which was higher than the previous findings that SCAs occur in approximately $0.3 \%$ of all live births [25]. A precise estimation of the detection rate of SCAs was not possible because most patients (4/5) with a high risk of SCAs declined the invasive test. As shown in the present study, the very low incidence of prenatal karyotyping for high-risk results of SCAs acquired by NIPT agreed very well with a previous study showing that $98.5 \%$ of pregnant women wanted to be informed if NIPT suspected an SCA, although only one third would consider amniocentesis [26]. The reason women stated for wanting to be informed was so that they could make informed choices and make preparations. According to the report by Jeon et al. [27], the low incidence of prenatal karyotyping was mostly due to the very high proportion of couples who were willing to continue the pregnancy after counseling, regardless of the fetal chromosomal status. Their willingness to continue pregnancy has been found to be significantly affected by the post-genetic counseling process and the genetic experience of the healthcare provider.

NIPT was requested for two main clinical indications: abnormal biochemical serum-screening results (54.3\%) and advanced maternal age (31.4\%). A total of 378 cases (378/910, 41.5\%) did not participate in screening tests before NIPT. In particular, 4 cases (0.4\%) were anxious, even though prior screening identified them as low-risk, and wanted to get tested nonetheless (Fig. 2). Many of them indicated that they would rather have an invasive test if NIPT was not available. Conventional screening tests were unable to alleviate participants' anxiety. In the present study, high-risk results of NIPT were detected in only $9(1.8 \%)$ out of 494 cases with abnormal biochemical serum-screening results. In general, patients identified as positive in the serum biochemical screening are subjected to extensive counseling and, as a result, often undergo invasive procedures. As most cases of positive results of biochemical screenings are false positives, such subsequent invasive testing creates undue anxiety for patients, increases medical costs, and places fetuses at risk for invasive procedure-related miscarriages. Sixteen cases (1.8\%) had high-risk NIPT results for aneuploidy in this study, indicating that approximately $98 \%$ of invasive diagnostic procedures can be avoided. Given a miscarriage rate of $0.5-2 \%$ of invasive procedures [28], NIPT saved the life of 3-10 healthy fetuses in this study. Another major advantage of NIPT is reporting the results as high- or low-risk, which makes it easier for parents to decide in favor of or against invasive testing [29]. However, NIPT did not diagnose aneuploidies other than trisomies 21, 18, and 13. Consequently, some women still desired a diagnostic test in order to exclude not only the three common trisomies, but also other fetal aneuploidies. In particular, invasive testing should be recommended in cases with high fetal nuchal translucency, even if NIPT identifies them as low-risk, because in such cases these trisomies account for only $75-80 \%$ of the associated clinically significant aneuploidies [30,31].

The limitations of this study were the insufficient followup regarding the NIPT results and the small sample size, which could introduce bias into the performance evaluation. In high- 
risk cases, only 7 out of 16 women (43.8\%) provided the results of a confirmatory diagnosis, mainly because the rest declined to provide clinical outcomes. This raised the importance of genetic counseling in the clinical application of NIPT and especially in avoiding potential misuse of NIPT as a diagnostic test. Before this test is widely adopted, both the clinicians and pregnant women should be fully aware that a high-risk result obtained by NIPT cannot be considered diagnostic and must be confirmed by karyotyping.

Undoubtedly, the main impact of the introduction of NIPT into clinical care has been a reduction in prenatal invasive diagnostic procedures and risk-free reassurances for women with lower false positive rates and higher positive predictive values than in current standard screening programs [32]. Recent studies $[10,15]$ have suggested that NIPT is a reliable method to use as a prenatal screening test for fetal aneuploidies. This study showed that MPS-based NIPT has high accuracy in detecting fetal chromosomal aneuploidies, and the introduction of NIPT as a prenatal screening test into clinical settings could prevent about $98 \%$ of unnecessary invasive diagnostic procedures. The challenge now is to develop a new algorithm for prenatal care and to integrate NIPT into national clinical guidelines to allow general access for all pregnant women who could benefit from NIPT. We hope that this study on NIPT will provide useful data for all healthcare personnel and further contribute to the fetal chromosomal abnormalities screening policy in Korea.

\section{Acknowledgements}

This study was supported by Seoul Clinical Laboratories Research Grant (2015, President: Kyoung-Ryul Lee).

\section{References}

1. Malone FD, Canick JA, Ball RH, Nyberg DA, Comstock CH, Bukowski $\mathrm{R}$, et al; First- and Second-Trimester Evaluation of Risk (FASTER) Research Consortium. First-trimester or second-trimester screening, or both, for Down's syndrome. N Engl J Med 2005;353:2001-11.

2. Kagan KO, Etchegaray $A$, Zhou Y, Wright $D$, Nicolaides KH. Prospective validation of first-trimester combined screening for trisomy 21. Ultrasound Obstet Gynecol 2009;34:14-8.

3. Nicolaides KH. Screening for fetal aneuploidies at 11 to 13 weeks. Prenat Diagn 2011;31:7-15.

4. Lo YM, Corbetta N, Chamberlain PF, Rai V, Sargent IL, Redman CW, et al. Presence of fetal DNA in maternal plasma and serum. Lancet 1997;350:485-7.
5. Chiu RW, Akolekar R, Zheng YW, Leung TY, Sun $H_{1}$ Chan KC, et al. Non-invasive prenatal assessment of trisomy 21 by multiplexed maternal plasma DNA sequencing: large scale validity study. BMJ 2011;342:c7401.

6. Ehrich M, Deciu C, Zwiefelhofer T, Tynan JA, Cagasan L, Tim R, et al. Noninvasive detection of fetal trisomy 21 by sequencing of DNA in maternal blood: a study in a clinical setting. Am J Obstet Gynecol 2011;204:205.e1-11.

7. Sparks $A B$, Struble $C A$, Wang $E T$, Song $K$, Oliphant A. Noninvasive prenatal detection and selective analysis of cell-free DNA obtained from maternal blood: evaluation for trisomy 21 and trisomy 18. Am J Obstet Gynecol 2012;206:319.e1-9.

8. Palomaki GE, Kloza EM, Lambert-Messerlian GM, Haddow JE, Neveux LM, Ehrich $M$, et al. DNA sequencing of maternal plasma to detect Down syndrome: an international clinical validation study. Genet Med 2011;13:913-20.

9. Chen EZ, Chiu RW, Sun H, Akolekar R, Chan KC, Leung TY, et al. Noninvasive prenatal diagnosis of fetal trisomy 18 and trisomy 13 by maternal plasma DNA sequencing. PLoS One 2011;6:e21791.

10. Bianchi DW, Platt LD, Goldberg JD, Abuhamad AZ, Sehnert AJ, Rava RP; MatErnal BLood IS Source to Accurately diagnose fetal aneuploidy (MELISSA) Study Group. Genome-wide fetal aneuploidy detection by maternal plasma DNA sequencing. Obstet Gynecol 2012; 119:890-901.

11. Norton ME, Brar H, Weiss J, Karimi A, Laurent LC, Caughey $A B$, et al. Non-Invasive Chromosomal Evaluation (NICE) Study: results of a multicenter prospective cohort study for detection of fetal trisomy 21 and trisomy 18. Am J Obstet Gynecol 2012;207:137.e1-8.

12. Guex N, Iseli C, Syngelaki A, Deluen C, Pescia G, Nicolaides $K H$, et al. $A$ robust second-generation genome-wide test for fetal aneuploidy based on shotgun sequencing cell-free DNA in maternal blood. Prenat Diagn 2013;33:707-10.

13. Palomaki GE, Deciu C, Kloza EM, Lambert-Messerlian GM, Haddow $J E$, Neveux LM, et al. DNA sequencing of maternal plasma reliably identifies trisomy 18 and trisomy 13 as well as Down syndrome: an international collaborative study. Genet Med 2012;14:296-305.

14. Nicolaides KH, Syngelaki A, Ashoor G, Birdir C, Touzet G. Noninvasive prenatal testing for fetal trisomies in a routinely screened firsttrimester population. Am J Obstet Gynecol 2012;207:374.e1-6.

15. Bianchi DW, Parker RL, Wentworth J, Madankumar R, Saffer C, Das AF, et al; CARE Study Group. DNA sequencing versus standard prenatal aneuploidy screening. N Engl J Med 2014;370:799-808.

16. American College of Obstetricians and Gynecologists Committee on Genetics. Committee Opinion No. 545: Noninvasive prenatal testing for fetal aneuploidy. Obstet Gynecol 2012;120:1532-4.

17. Devers PL, Cronister A, Ormond KE, Facio F, Brasington CK, Flodman 
P. Noninvasive prenatal testing/noninvasive prenatal diagnosis: the position of the National Society of Genetic Counselors. J Genet Couns 2013;22:291-5.

18. Benn P, Borrell $A$, Cuckle $H$, Dugoff $L$, Gross $S$, Johnson JA, et al. Prenatal detection of Down syndrome using massively parallel sequencing (MPS): a rapid response statement from a committee on behalf of the Board of the International Society for Prenatal Diagnosis, 24 October 2011. Prenat Diagn 2012;32:1-2.

19. Morgan S, Delbarre A, Ward P. Impact of introducing a national policy for prenatal Down syndrome screening on the diagnostic invasive procedure rate in England. Ultrasound Obstet Gynecol 2013;41:5269.

20. Muller PR, Cocciolone $R$, Haan EA, Wilkinson $C$, Scott $H$, Sage $L$, et al. Trends in state/population-based Down syndrome screening and invasive prenatal testing with the introduction of first-trimester combined Down syndrome screening, South Australia, 1995-2005. Am J Obstet Gynecol 2007;196:315.e1-7.

21. Musci TJ, Fairbrother G, Batey A, Bruursema J, Struble C, Song K. Noninvasive prenatal testing with cell-free DNA: US physician attitudes toward implementation in clinical practice. Prenat Diagn 2013;33: 424-8.

22. Chetty S, Garabedian MJ, Norton ME. Uptake of noninvasive prenatal testing (NIPT) in women following positive aneuploidy screening. Prenat Diagn 2013;33:542-6.

23. Dan $S$, Wang $W$, Ren J, Li $Y, H u H, X u$ Z, et al. Clinical application of massively parallel sequencing-based prenatal noninvasive fetal trisomy test for trisomies 21 and 18 in 11,105 pregnancies with mixed risk factors. Prenat Diagn 2012;32:1225-32.

24. Committee Opinion Summary No. 640: Cell-free DNA screening for fetal aneuploidy. Obstet Gynecol 2015;126:691-2.
25. Morris JK, Alberman $E$, Scott $C$, Jacobs $P$. Is the prevalence of Klinefelter syndrome increasing? Eur J Hum Genet 2008;16:163-70.

26. Lau TK, Chan MK, Salome Lo PS, Chan HY, Chan WK, Koo TY, et al. Non-invasive prenatal screening of fetal sex chromosomal abnormalities: perspective of pregnant women. J Matern Fetal Neonatal Med 2012;25:2616-9.

27. Jeon KC, Chen LS, Goodson P. Decision to abort after a prenatal diagnosis of sex chromosome abnormality: a systematic review of the literature. Genet Med 2012;14:27-38.

28. Alfirevic Z, Sundberg K, Brigham S. Amniocentesis and chorionic villus sampling for prenatal diagnosis. Cochrane Database Syst Rev 2003;(3):CD003252.

29. Nicolaides KH, Chervenak FA, McCullough LB, Avgidou K, Papageorghiou A. Evidence-based obstetric ethics and informed decision-making by pregnant women about invasive diagnosis after first-trimester assessment of risk for trisomy 21. Am J Obstet Gynecol 2005;193:322-6.

30. Snijders RJ, Noble P, Sebire N, Souka A, Nicolaides KH. UK multicentre project on assessment of risk of trisomy 21 by maternal age and fetal nuchal-translucency thickness at 10-14 weeks of gestation. Fetal Medicine Foundation First Trimester Screening Group. Lancet 1998; 352:343-6.

31. Chitty LS, Kagan KO, Molina FS, Waters JJ, Nicolaides KH. Fetal nuchal translucency scan and early prenatal diagnosis of chromosomal abnormalities by rapid aneuploidy screening: observational study. BMJ 2006;332:452-5.

32. Norton ME, Jacobsson $B$, Swamy GK, Laurent LC, Ranzini AC, Brar $H$, et al. Cell-free DNA analysis for noninvasive examination of trisomy. N Engl J Med 2015;372:1589-97. 